Vol. 5, n`2 | 2001

Varia

\title{
«La lie de la canaille ». Larrons, brigands et filous de profession : la répression du banditisme à Genève (1682-1792)
}

Christophe Dubied

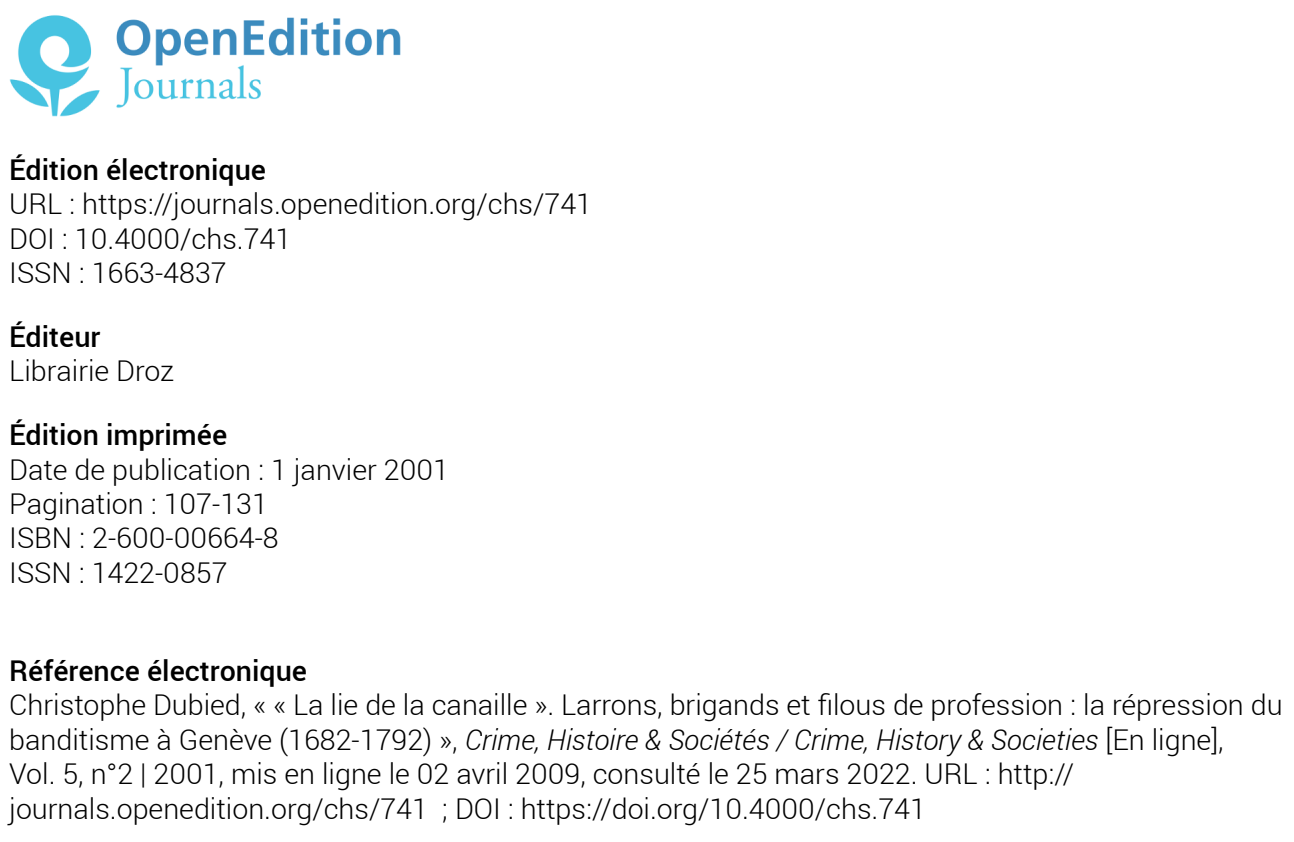




\title{
«La lie de la canaille» Larrons, brigands et filous de profession: la répression du banditisme à Genève (1682-1792)
}

\author{
Christophe Dubied'
}

\begin{abstract}
Fondé sur l'étude des procès criminels, cet article analyse les différentes circonstances aggravantes des délits commis par des criminels associés, dont le cumul détermine la dangerosité particulière du banditisme. Il décrit l'univers des bandes qui agissent à Genève et dans ses campagnes (organisation, profil des bandits et des complices, mobilité), et discute des multiples enjeux et difficultés de la répression pénale (entraide judiciaire entre États, instruction des procès, châtiment des crimes) de ce type particulier de criminalité, dans une République au territoire morcelé et exigu.

Based on study of criminal proceedings, this article analyses the different aggravating circumstances connected with crimes committed by associated offenders, the combination of which determines the specific dangerousness of banditry. The world of gangs active in Geneva and the surrounding countryside is described (organisation, profile of bandits and their accomplices, mobility). The numerous debates and difficulties involved in prosecution of this specific kind of criminality in a small Republic whose territory was divided into many jurisdictions are discussed (international judicial cooperation, conduct of investigations, punishment of crimes).
\end{abstract}

À Jitka...

\footnotetext{
Te l'ai déjà puisée dans ses propres réponses, cette information de sa vie, si honteuse pour lui et si triste pour ses juges. C'est là que Vos Seigneuries ont vu où, avec qui et de quoi il a vécu. C'est là qu'il se peint ou comme assassin, ou comme calomniateur, et qu'il décèle par une multitude de traits la férocité de son âme. C'est là qu'occupé sans cesse à égarer les magistrats informateurs par une suite de réticences, de mensonges et de contradictions, il ne rend gloire à la vérité qu'à la dernière extrémité. Et que ne témoignant ni repentir ni remords, il ne permet pas même à ses juges de chercher à le sauver par l'idée d'une régénération, dont les tribunaux doivent toujours avoir l'espoir, tant que cette espérance n'est pas démontrée chimérique.

Ainsi, quelque douloureux qu'il soit de prononcer un arrêt de mort, la sévérité du ministère que je remplis aujourd'hui me fait un devoir pénible mais

1 Christophe Dubied est licencié en lettres de l'Université de Genève (1999). Il est actuellement administrateur de la mission de Médecins sans Frontières en Afghanistan. Cet article synthétise un mémoire de licence intitulé: "La lie de la canaille». Larrons, brigands et filous de profession: la répression du banditisme à Genève (1682-1792), Université de Genève, Faculté des Lettres, Département d'Histoire générale, Unité d'Histoire moderne, juin 1999, direction M. Porret; 175 fol. dactylographiés.
} 
nécessaire de me prévaloir de toute la rigueur de la loi pour demander que Vos Seigneuries délivrent la société d'un malfaiteur qui, associé depuis longtemps avec les brigands de nos environs, loin de paraitre disposé à renoncer à ce gente de vie, se peint lui-même à chaque ligne de ses réponses comme capable des mêmes forfaits qu' eux $^{2}$.

Scélérats, filous, larrons et brigands ont de tous temps effrayé autant que fasciné leurs contemporains: preuves en sont les multiples fictions littéraires, théâtrales et plus récemment cinématographiques réalisées à leur sujet à travers les siècles. Ces histoires plurielles de figures devenues légendaires, images de personnages réels revisitées et transfigurées par la fiction, sont entrées dans l'histoire au singulier par le biais de toute une série de travaux entamés à partir des années 1960. Emboîtant le pas à Eric John Hobsbawm, qui proposa le premier une grille de lecture du banditisme considéré comme phénomène de société, plusieurs historiens se sont penchés sur la figure du «bandit social», obscur personnage devenu criminel par nécessité, «paysan hors-la-loi que le seigneur et l'État considèrent comme un criminel, mais qui demeure à l'intérieur de la société paysanne, laquelle voit en lui un héros, un champion, un vengeur, un justicier[...]» ${ }^{3}$.

Même si quelques aspects de la criminalité associative genevoise au XVIII siècle ont déjà été partiellement étudiés ${ }^{4}$, aucune recherche spécifiquement consacrée au banditisme n'avait encore vu le jour. Pourtant, les sources de la pratique pénale révèlent une spécificité de la criminalité associative genevoise au XVIII ${ }^{\mathrm{e}}$ siècle, qui justifie une telle étude. En effet, à côté du modèle désormais classique du «banditisme social», caractérisé par le vol avec violence en milieu rural (vol de grand chemin, attaque de maisons isolées), dont les protagonistes sont issus principalement des campagnes frontalières de la Savoie et du Pays de Gex, se développe au XVIII ${ }^{e}$ siècle une nouvelle forme de criminalité associative de plus en plus active à l'intérieur de la cité.

Parallèlement aux troupes de brigands qui continuent sporadiquement de semer la terreur dans les campagnes genevoises, apparaissent des bandes spécialisées dans les vols avec effraction et les filouteries. L'originalité de ces associations de «scélérats de profession ", tient aussi bien aux types de crimes qu'elles commettent, qu'à la complexité de leurs réseaux de complicités, à leur professionnalisme, aux trajectoires personnelles des bandits (caractérisées par la récidive et "l'endurcissement dans le crime»), et aux difficultés qu'elles posent à l'appareil répressif chargé de poursuivre pénalement leurs actes.

2 AEG, série 1, PC 15159, 1787, vols et assassinats, "Conclusions », folio 49. Nos sources: Procès criminels, série 1 (dorénavant $P C$ ), proviennent des Archives d'État de Genève (AEG); nous mentionnons le numéro de la procédure ou de l'information, l'année de son ouverture, ainsi que le chef d'accusation figurant sur le «Registre des procès et informations instruits entre 1396 et 1817" (5 vol.). Nous avons modernisé la leçon de nos sources manuscrites.

3 Hobsbawm $(1972$, p. 8). Bien qu'Hobsbawm fut le premier à avoir proposé un modèle d'analyse du banditisme, il faut mentionner les travaux antérieurs de F. Funck-Brentano (1924), qui catalogue les différentes formes que revêt le banditisme à travers les âges. Parmi les nombreux travatıx consacrés à la criminalité d'Ancien Régime, qui font une part plus ou moins grande à l'étude du banditisme, citons, entre autres, ceux de F. Egmond pour les Pays-Bas, J.-M. Beattie pour l'Angleterre, N. Castan pour la France, M. Porret pour Genève.

$4 \quad$ Cf. Porret (1995a, pp. 297-339). 
Le terme de banditisme, que nous utilisons par commodité, n'apparaît dans la langue française qu' au XIX ${ }^{\mathrm{e}}$ siècle, et recouvre de multiples chefs d'accusation, tels que brigandage, rébellion, filouterie, larcin, vol, assemblée illicite, complicité d'une bande de voleurs, recel, ou encore assassinat et meurtre. L'imprécision de la casuistique judiciaire exige de recourir à une définition plus générale du banditisme, fondée sur l'atrocité des crimes, elle-même déterminée, selon le droit criminel d'Ancien Régime, par l'analyse des circonstances qui entourent l'acte délictueux ${ }^{5}$. Le cumul des circonstances de l'association et de la récidive, ainsi que de circonstances matérielles très aggravantes (vol nocturne, violence, port d'armes, etc.) caractérise le banditisme et détermine, aux yeux des procureurs généraux, sa dangerosité ${ }^{6}$ particulière. Le cumul des circonstances aggravantes a servi ici de critère de sélection de soixante procédures et informations judiciaires, intentées contre des bandes de criminels récidivistes coupables de vols qualifiés (filouterie, effraction, fausses clefs, vol de grand chemin, brigandage) et/ou de crimes de sang (excès, violences, assassinat, meurtre) entre 1682 et $1792^{7}$. La doctrine pénale (ouvrages de jurisprudence et de commentaire des lois criminelles, principalement d'origine française) a permis de replacer dans le contexte juridique de la répression pénale d'Ancien Régime les réflexions et les raisonnements des procureurs généraux lors de la qualification des crimes ${ }^{8}$.

\section{LE CUMUL DES «CIRCONSTANCES AGGRAVANTES»}

\section{Les «plus atroces de tous les vols»}

Le banditisme, quelle que soit la forme qu'il prenne, «accumule les circonstances aggravantes de nombreux délits simples et qualifiés ${ }^{9}$. En témoigne, par exemple, le vol perpétré à l'encontre du Sieur Foulquier dans sa maison sise aux alentours de Lausanne au mois de juillet 1783 , raconté ici par un de ses auteurs lors de son interrogatoire:

Qu'ils se réunirent tous environ à huit heures du soir à demie lieue d'ici sur la route, ayant bu un coup à l'auberge de l'Écu à Lutry, savoir Lyonnais, Creuset et lui détenu, en attendant Blanchard et Favret, et qu'ils se joignirent avec ces deux

\section{Cf. Porret (1995a).}

6 Le terme de dangerosité, apparu dans la langue française en 1963, a été élaboré et finalisé par la criminologie contemporaine. Nous l'utilisons ici, par commodité, à des fins descriptives.

7 Les sources ont été sélectionnées selon le nombre de personnes impliquées (trois au minimum, à l'exception de quelques procédures ouvertes contre un seul criminel, par ailleurs connu comme faisant partie d'une bande), et selon les indices, plus ou moins explicites, désignant l'un des inculpés au moins comme récidiviste notoire. Sur la soixantaine de procédures et informations retenues, une dizaine de gros procès contient un réquisitoire, soit «Conclusions» du procureur général de la République. Les affaires de contrebande, de fausse monnaie, de sédition populaire ou de complot politique, ou encore les vols de peu d'importance commis, par exemple, par les ouvrières d'un atelier ou par des délinquants juvéniles, n'ont pas été retenues.

8 Dès le XVI siècle, la procédure criminelle genevoise est inquisitoire; à propos des spécificités de ce type de procédure, cf. Dyonet (1987). Les Édits civils de la République de Genève, promulgués en $1568 \mathrm{et}$ revus en 1713 , ne fixent que très sommairement le cadre de la procédure criminelle, et l'essentiel des règles qui la régissent proviennent de l'Ordonnance criminelle de 1670 de Louis XIV.

9 Porret (1995a, p. 338). 
derniers à la sortie du village de Pully. [...] Qu'alors Blanchard, ayant trouvé une échelle ne sachant où, il l'appuya contre le mur et travailla à enlever un carreau de la fenêtre, opération qui lui prit beaucoup de temps. [...]Que Blanchard entra le premier et alluma une chandelle; qu'ensuite Lyonnais entra aussi, que ces deux restèrent longtemps et que Favret entra le dernier, Bressan ou Creuset étant resté en bas en faction [...].

Qu'ils avaient déterminé entre eux de ne point faire de mal à Monsieur Foulquier, mais de le lier pour qu'il ne pût pas traverser leur entreprise, ce qu'ils exécutèrent. [...]

Qu'ils apportèrent chacun des parties d'argenterie dans le jardin de devant la maison, de là dans un verger vis-à-vis, qu'ils en mirent autant qu'ils purent dans le sac de lui Comtois à qui ils le remirent [...]. Que dans ces entrefaites, la cloche de Monsieur Foulquier ayant sonné avec beaucoup de force, ils se sauvèrent les uns deçà, les autres delà, et lui détenu avec son $\operatorname{sac}^{10}$.

Commis nuitamment par des criminels récidivistes associés, avec escalade, effraction, port d'armes et préméditation, portant en outre sur une quantité considérable de biens meubles, ce vol est qualifié par plusieurs circonstances aggravantes, qui, selon les principes juridiques du droit criminel d'Ancien Régime, en augmentent la gravité, et par conséquent la peine. Les vols faits «avec effraction, port d'armes et violence publique », tel que celui-ci, sont d'après le jurisconsulte français Daniel Jousse (1704-1781), les «plus atroces de tous les vols $»^{11}$. Tous les actes de banditisme que nous avons recensés (à l'exception des vols simples) sont qualifiés par plusieurs circonstances aggravantes, en premier lieu les circonstances dites matérielles. Celles-ci « se tirent ou de la MANIERE dont le Vol a été fait, comme s'il est commis avec effraction, avec armes, ou avec déguisement, ou bien par adresse \& filouterie; [...] ou de la QUALITÉ de la chose volée [...]; ou de la QUANTITÉ de la chose volée, comme si le vol est considérable [...]; ou du LIEU, comme si c'est à l'Église, dans les Maisons royales, au Palais ou Auditoire de la Justice, dans les Spectacles publics, sur les grands chemins; ou du TEMPS, comme s'il est fait pendant la Nuit $[\ldots] \gg{ }^{12}$.

Les circonstances matérielles du crime, plus ou moins aggravantes selon qu'il s'agit d'un vol de grand chemin ou d'un vol à l'étalage, s'accompagnent toujours de deux autres circonstances inhérentes à la qualité et au nombre des criminels: la récidive et l'association. Le procureur général Des Arts, par exemple, relève dans son réquisitoire contre une bande de criminels coupables d'avoir volé plusieurs ballots de textiles à deux maisons de commerce en se déguisant en voituriers en 1774, que l'association de «filous de profession» s'ajoute aux circonstances matérielles du crime pour en aggraver la qualification:

Ces vols sont donc qualifiés par la grandeur de leur objet, et par l'adjonction du crime de faux. Ils le sont encore par l'association, soit espèce d'attroupement qui a eu lieu pour le commettre, par la qualité des personnes qui sont entrées dans cette association, qui sont des déserteurs, des gens sans aveu, des filous de profession ${ }^{13}$.

10 AEG, série 1, PC 14236, 1784, vols avec effraction, «Confessions de Comtois le Rouge», folios 1-4.

Jousse (1771, IV, p. 222).

Muyart de Vouglans (1757, pp. 557-558).

13 PC 12668, 1775, vols et complicités, «Conclusions», folios 9-10. 
$\mathrm{Au} \mathrm{XVII}^{\mathrm{e}}$ siècle, l'immense majorité des crimes commis en association et jugés à Genève consiste en actes de brigandage, accompagnés de violences physiques: plus d'une procédure sur deux comprend le chef d'accusation d'assassinat ou de meurtre. Au cours du siècle suivant, on assiste à une forte diminution des diverses formes de brigandage (dont le vol de grand chemin ne représente qu'un tiers, le reste se partageant entre l'attaque de maisons isolées et le rançonnement), exercées par des troupes de bandits armés dans les campagnes, qui ne représentent plus que $15 \%$ de la totalité des crimes commis par des bandits.

La proportion de crimes de sang perpétrés dans le cadre de la criminalité associative suit la même évolution. Règlements de comptes entre bandits, vengeances exercées à l'encontre de délateurs, plus rarement violences commises au cours même d'un vol, les crimes de sang constituent pour ainsi dire des charges collatérales aux actes de banditisme. Les onze assassinats recensés entre 1682 et 1792, additionnés aux batteries et autres excès, ne forment que $12 \%$ de l'ensemble des crimes imputés à des bandits. Ces chiffres témoignent d'un «affaissement séculaire de la criminalité de sang qualifiée par des 'circonstances atroces' ${ }^{14}$ à Genève au XVIII ${ }^{\mathrm{e}}$ siècle, comme dans de nombreuses régions d'Europe.

En revanche, les crimes contre les biens perpétrés en association dans le cadre urbain se multiplient, en premier lieu les vols avec effraction ou fausses clefs, qui représentent $39 \%$ des chefs d'accusation dont sont chargés des bandits. Les filouteries en tous genres, du vol à la tire au vol à l'étalage, en passant par le vol astucieux commis avec tromperie ou déguisement, représentent, quant à eux, 12\% de la totalité des crimes recensés ${ }^{15}$.

L'augmentation des crimes contre les biens au détriment de la criminalité de sang ne peut se comprendre que dans son rapport à l'évolution globale des structures sociales et économiques. La République de Genève bénéficie, au cours du siècle, d'une prospérité économique exceptionnelle: les manufactures d'horlogerie et d'indiennes, les établissements bancaires prospèrent. Parallèlement, la population s'accroît de près de $60 \%$ entre 1711 et $1792^{16}$. La forte croissance de la ville tout au long du siècle, lieu de concentration à la fois des richesses et des surplus démographiques des campagnes attirés par la nouvelle demande de main-d'œuvre des manufactures en plein développement, s'accompagne d'une modification structurelle de la criminalité. L'augmentation des richesses disponibles et la croissance du volume des échanges incitent au vol; parallèlement, les autorités font preuve d'une sensibilité plus grande aux atteintes contre la propriété privée. Le banditisme trouve évidemment là un terrain d'activité privilégié: «il se déploie dans les villes», écrit Nicole Castan «avec un succès d'autant plus redoutable qu'il y rencontre un cadre et des ressources plus stimulantes» que dans les campagnes. «Refuge mais mirage, c'est dans ce sens que la ville est franchement criminogène, étalant scandaleusement,

14 Berchtold, Porret (1994, p. 52). À propos de l'évolution des formes de la criminalité au XVIII siècle, et plus particulièrement le passage de la violence au vol, $c f$. Weisser (1982) et Johansen, Stevnsborg (1986). Certaines régions, cependant, ne suivent pas cette évolution; tel est le cas, par exemple, de la principauté de Neuchâtel, comme l'a montré Henry (1984).

15 Le calcul de ces chiffres est fondé sur un total de 147 crimes recensés dans la période qui nous intéresse. Les $22 \%$ non mentionnés concernent soit des vols simples, commis par un bandit en solitaire (vol de pain à une devanture de boulangerie, ou d'une pièce d'uniforme dans un jardin, par exemple), soit des crimes qu'aucune information précise ne permet de classer dans l'une ou l'autre catégorie.

16 Ces chiffres sont cités par Dufour (1997, p. 89). 
comme elle le fait dans ses boutiques et sur ses marchés, l'abondance de sa marchandise; société de convoitise où une répression hasardeuse encourage à voler ${ }^{17}$.

\section{La "carrière du crime"}

François Rosay, originaire de Lugrin en Savoie, n'a que 22 ans lorsqu'il est exécuté en 1787 pour différents vols et assassinats commis sur tout le pourtour du bassin lémanique. Dans son réquisitoire, le procureur général Naville retrace les différentes étapes de son «endurcissement dans le crime», telles qu'elles ressortent des confessions de l'accusé et des témoignages recueillis au cours de l'instruction du procès:

[S]a vie est plus remplie d'événements que ne le serait celle d'un scélérat qui aurait vieilli dans le crime. [...] Dès sa première jeunesse il a été enclin au vol; les exhortations et les châtiments de Pierre Rosay son père n'ont pu le ramener: il a bientôt quitté la maison paternelle, et il paraît même que son premier départ fut la dernière tentative de sa famille pour le ranger à son devoir. Car son frère aîné et lui s'engagèrent ensemble au Régiment de Savoie, où cet aîné est resté, et avait intention de le retenir; tandis que le prévenu, désertant de ce régiment peu après s'y être engagé, est revenu au pays commettre les excès et les brigandages dont Vos Seigneuries vont être occupées.

Il a porté huit noms différents (de son propre aveu) dans les divers lieux où ils s'est montré, et il en a changé trois fois dans les prisons.

[...] II s'est donné en divers temps six patries différentes et trois dans vos prisons.

[...] Les divers métiers qu'il a exercés ou prétendu exercer sont sans nombre.

[...] Il existe au procès comme bien prouvées trois désertions.

[...] Il a été déjà quatre fois repris par justice [...].

Sa vie, depuis quelques temps, était de passer le jour dans des bois ou dans des lieux écartés [...]. Il sortait de là la nuit pour faire ses coups de main. [...] Mais ce qui a été relevé suffit amplement pour constater que cet homme menait la vie d'un brigand dans toute l'étendue du terme ${ }^{18}$.

Les faits mentionnés ci-dessus, minutieusement compilés par la partie publique, aggravent les charges qui pèsent sur Rosay. En effet, le droit criminel d'Ancien Régime pose comme principe que la qualification du vol dépend, entre autres, de la qualité de la personne qui l'a commis: la qualification augmente si cette personne est un déserteur ou un vagabond. L'habitude agit dans le même sens: si le voleur est un récidiviste, le crime s'en trouve aggravé. Ceci explique pourquoi les procureurs généraux passent systématiquement en revue les antécédents biographiques et judiciaires des prévenus avant de qualifier les crimes et de requérir les peines: «résultat de l'effort d'une justice qui veut retrouver une logique et une cohérence du comportement criminel tout au long de l'existence du prévenu ${ }^{19}$, la biographie criminelle répond à la nécessité de déterminer avec exactitude les circonstances individuelles du crime et de prouver l'incorrigibilité du délinquant. Celle de Rosay présente tous les traits de l'endurcissement dans le crime. Plusieurs fois repris de justice, déser-

\footnotetext{
17 Castan (1980, pp. 278 et 290).

18 PC 15312/15313, 1787, vols, violences, assassinats, «Conclusions», folios 1-6.

19 Geremek (1980, p. 42). En ce qui concerne la forme et les implications idéologiques de la biographie criminelle: $c f$. Porret (1995b).
} 
teur, Rosay n'avoue aucun domicile fixe, ni aucune profession crédible. Dans les termes juridiques de l'époque, il est une personne «sans aveu», c'est-à-dire que sa qualité le rend d'emblée suspect et est retenue en tant que circonstance aggravante de ses crimes, dès lors que ceux-ci sont prouvés.

Les circonstances individuelles, qui signalent l'endurcissement de Rosay dans le crime et aggravent la qualification de ses délits se retrouvent, à peu de choses près, dans toutes les biographies des «plus scélérats» d'entre les prévenus incriminés dans les affaires de banditisme. Sur l'ensemble des prévenus, 55 individus présentent une ou plusieurs des caractéristiques - désertion, passage aux galères, récidive, absence d'aveu qui en font des «scélérats de profession» aux yeux de la justice ${ }^{20}$. Ces criminels professionnels forment pour ainsi dire le noyau, la pierre angulaire des bandes de voleurs : ce sont eux qui planifient les vols, qui disposent des connaissances et des appuis nécessaires à leur réalisation, eux enfin, qui écopent des peines les plus sévères, en raison de leurs antécédents judiciaires et de leur participation active aux crimes.

Dans leur entourage gravitent les autres membres de la bande - complices, femmes ou concubines, receleurs, cabaretiers - qui leur fournissent aide et soutien dans la réalisation de leurs crimes. À l'image d'une confrérie professionnelle, l'association criminelle, grâce à la division des tâches et à la solidarité de ses membres, permet aux voleurs d'augmenter leurs chances de succès, et donc de profit, tout en diminuant les risques, répartis entre tous. Une grande partie des criminels, après leurs débuts dans la «carrière du crime», optent pour le regroupement en bande. Les contacts noués dans les prisons avec les gens du «milieu» favorisent les projets de vol en commun: de simple voleur, le criminel devient bandit. Les vols de grande envergure, exigeant des moyens adaptés à la difficulté de l'entreprise, succèdent aux premiers illégalismes commis en solitaire.

\section{Un «tas de vauriens très dangereux»}

Pour la justice, l'association criminelle constitue une circonstance très aggravante qui, cumulée aux autres, augmente la qualification du crime. En effet, la répartition des tâches et des responsabilités qui caractérise le fonctionnement des bandes de voleurs, ainsi que leurs vastes réseaux de complicités, augmentent la difficulté des poursuites pénales et permet des vols de grande envergure. Dans ses "Conclusions» contre une partie des membres de la Grande Bande en 1784, le Procureur Naville relève avec insistance le danger que représentent de telles associations pour la société:

Là partie publique observe comme une qualification grave des délits actuellement sous vos yeux, M. S., qu'ils ont tous été commis par des voleurs associés. Que

20 La majorité de ces bandits a entre 20 et 40 ans, la moyenne se situant à 31 ans. Leur nationalité est très nettement étrangère à Genève: on ne compte que deux natifs genevois sur 55 . Plus de la moitié d'entre eux vient des pays français proches de Genève (Pays de Gex, Dauphiné, Franche-Comté), un quart de Savoie, et le reste du Piémont, de la Principauté de Neuchâtel et d'Allemagne. D'un point de vue socio-professionnel, le groupe le plus nombreux est formé d'artisans de toutes professions: tailleur, chaudronnier, barbier, boucher, chapelier, horloger, meunier, orfèvre, etc. Quatre bandits font état du métier de marchand-colporteur; quatre autres sont soldats de la garnison de Genève. Plusieurs ouvriers, un écrivain, un mendiant, un laboureur et, notable exception, un noble savoyard complètent le tableau. 
cette association, par les ressources des voleurs, leurs connaissances, leurs moyens de cacher les vols, de les écouler, d'en préparer de nouveaux, était la plus alarmante qu'il fût possible d'imaginer, la plus nuisible à la sécurité publique ${ }^{21}$.

Selon les doctrinaires du droit criminel d'Ancien Régime, le nombre de personnes requis pour former une association criminelle n'est pas clairement défini: «les uns exigent celui de huit, d'autres de quatre ou cinq seulement», écrit Serpillon $(1695-1772)^{22}$. En définitive, bien que les jurisconsultes s'accordent à fixer le nombre minimal à quatre personnes, celui-ci dépend en dernier lieu «des circonstances, et de la prudence des juges ", seuls habilités à retenir ou non le chef d'association $^{23}$. Les bandes criminelles ${ }^{24}$ qui sévissent à Genève au XVIII ${ }^{e}$ siècle disposent en général d'une organisation poussée et de réseaux de soutien dépassant largement les frontières de la République. Les membres de ces bandes se rassemblent ponctuellement pour réaliser leurs coups, puis se séparent, avant de se retrouver plus tard pour une nouvelle entreprise. Autour des «chefs» gravitent plusieurs complices, recrutés à l'occasion d'un vol ou familiers des bandits qui les suivent dans leurs déplacements ${ }^{25}$. Couturières ou servantes, mendiants recrutés pour l'occasion, jeunes gens sans emploi ou souffrant d'un handicap, ces auxiliaires fournissent de précieux renseignements sur les vols, en espionnant les lieux, en décrivant les habitudes d'une maisonnée, ou encore en évaluant la quantité du butin potentiel. Un jeune homme affligé de surdi-mutité, arrêté en 1787 en compagnie de deux autres complices, sert, par exemple, d'indicateur à ses peu recommandables compagnons: son handicap, allié à «une intelligence bien au-dessus de ses ressources» lui ont, selon le procureur général subrogé Rigaud, "procuré l'entrée dans la bande de voleurs qui l'a agrégé comme un espion précieux qui pourrait la précéder dans les maisons sans exciter la défiance ${ }^{26}$.

Après le vol, les receleurs fournissent aux fugitifs le refuge de leurs auberges (appelées tapis francs en argot) et usent de leurs relations commerciales pour écouler le butin. David Manilly, condamné en 1781 au bannissement perpétuel pour recel, est décrit par le procureur général Butini comme «l'homme d'affaires des

21 PC 14236, vols avec effraction, «Conclusions», folio 46.

22 (1767, I, p. 92). Les doctrinaires utilisent de préférence les termes «attroupement» ou "assemblée illicite » lorsqu'ils traitent de l'association criminelle; ce dernier terme est en revanche majoritairement utilisé par les procureurs dans leurs réquisitoires.

23

24

Jousse (1771, IV, p. 64).

Nous avons dénombré, sur l'ensemble du siècle, une vingtaine de bandes, dont l'existence est mentionnée dans les pièces de procès. Il faut cependant prendre ce chiffre avec précaution, puisque plusieurs de ces bandes ne sont que signalées sans que leurs actes ne fassent l'objet d'une enquête. Qui plus est, ces bandes se font et se défont au gré des occasions de vol et des rencontres, des arrestations et des condamnations.

La moitié des complices inculpés dans les affaires de banditisme sont des femmes, généralement les épouses ou concubines des bandits, dont l'origine sociale et géographique est similaire à celle de leurs compagnons. Leur répartition dans les classes d'âge présente un large éventail qui va de 15 à 52 ans, avec une moyenne situé à 29 ans. Les complices de sexe masculin, eux aussi d'origine étrangère à une large majorité, sont généralement plus jeunes que les bandits: à l'exception d'un vieillard de 70 ans, tous ont entre 14 et 36 ans, la moyenne se situant autour de 25 ans. À tous ces déracinés, peu ou pas qualifiés professionnellement, l'association criminelle offre une protection et un réseau de solidarités qui leur permettent de survivre.

PC 15159, vols et assassinats, «Conclusions», folio 60. 
voleurs, $[\ldots]$ une espèce de centre où viennent aboutir les voleurs et les vols $»^{27}$. Aubergistes, marchands ambulants ou encore orfèvres, les receleurs disposent en général d'une stabilité professionnelle et d'un domicile fixe: ils servent en quelque sorte de relais entre le monde des criminels et la société dans laquelle ils sont intégrés.

Le nombre des individus composant les bandes jugées à Genève varie énormément. Il est très difficile d'avancer des chiffres précis: seule une évaluation approximative peut être faite, à partir des témoignages des inculpés, qui dénoncent d'autres complices non mentionnés au procès. Le nombre maximal d'inculpés appartenant à la même bande (par ailleurs jugés dans deux procédures successives) atteint 21 : il s'agit des membres de la «Grande Bande», ainsi dénommée par le procureur général Naville, dont les deux procès se déroulent à Genève en 1783 et $1784^{28}$. Si l'on prend en compte tous les complices dénoncés par les prévenus appartenant à cette bande, y compris les femmes, le chiffre s'élève à 73 personnes. Dans deux autres affaires, le nombre total des bandits inculpés ou nommés au procès, receleurs compris, dépasse la trentaine ${ }^{29}$. La plupart du temps, pourtant, le nombre n'excède pas dix personnes; rien de comparable, donc, avec les bandes, composées de plus d'une centaine d'individus, menées par Mandrin ou Cartouche en France, ni avec les grandes troupes de gitans écumant les campagnes des Pays-Bas, décrites par Florike Egmond $^{30}$.

Le professionnalisme des bandits, allié à une grande mobilité géographique et au soutien de vastes réseaux de complicités, leur permet bien souvent d'échapper aux châtiments qu'il encourent. Située au centre d'un territoire exigu et morcelé, l'opulente cité de Calvin, avec ses ateliers d'horlogerie et de bijouterie, ses manufactures textiles, constitue un terrain de prédilection pour les bandes de voleurs. Bordé au Nord par le Pays de Gex français, par la Savoie à ses autres frontières, distant de quelques kilomètres seulement du Pays de Vaud et disposant d'une ouverture sur le lac, le petit territoire genevois offre de multiples possibilités de fuite à ceux qui doivent $s$ 'en éloigner rapidement. La petite ville sarde de Carouge, sise juste à l'extérieur des murailles, le grand chemin qui mène à Lausanne, la route d'Évian et le lac sont autant d'échappatoires offertes aux voleurs. Les réseaux de complicité des bandits forment de gigantesques toiles d'araignée dont les fils s'étendent bien au delà des frontières de la République. Les auberges où les bandits fomentent leurs coups, trouvent refuge dans leur fuite et écoulent le produit de leurs entreprises illicites, forment les points d'ancrage de ces réseaux de complicités transfrontaliers.

Les deux procès, particulièrement bien documentés, intentés contre la Grande Bande en 1784, fournissent des renseignements très détaillés sur le réseau de complicités mis en place par les bandits: celui-ci couvre un espace s'étendant du Dauphiné à la région neuchâteloise, au Pays de Vaud et à toute la région lémanique, à la Savoie et au Piémont. Lors des aveux faits avant sa condamnation à la peine

27 PC 13630, complicité, liaisons suspectes, «Conclusions», folio 10. Dix receleurs sur les quatorze inculpés dans des affaires de banditisme au XVIII' siècle sont de sexe masculin; leur moyenne d'âge avoisine les 40 ans; à l'exception d'un bourgeois de Genève, ils sont tous d'origine étrangère.

28 Il faut encore ajouter à ce chiffre les deux complices jugés à la même époque à Lausanne par la justice vaudoise; c $f$. PC 14236, 1783; PC 14236bis, 1784; PC 14202, 14218, 14323, 14288, 14407 et 14447 , qui concernent la même affaire.

29 PC 9710, 1750, vols, brigandages et PC 15312/313, 1787, vols, violences, assassinats.

30 Cf. Funck-Brentano (1924); Egmond (1993). 
capitale en 1784, Comtois le Rouge donne les noms de plus de dix cabaretiers et aubergistes qui logent les voleurs et recèlent leur butin. Les auberges de ces « tapissiers francs » forment, selon les termes du procureur Naville « comme une espèce de chaîne dont les deux extrémités se perdent en Valais et en Piémont ${ }^{31}$. Le report des lieux de recel sur une carte géographique, en regard des lieux où la bande commet ses vols, montre que chaque région où elle est active abrite un ou plusieurs receleurs vers lesquels convergent les bandits avec leur butin. Ces auberges se trouvent toujours sur des voies de communication importantes entre les principales villes de Suisse romande et de Savoie, ou dans ces villes mêmes, chemins que les bandits empruntent lorsqu'ils se retirent d'un endroit après y avoir commis un vol.

Caractéristique de la criminalité associative, la mobilité répond à la nécessité d'échapper aux poursuites, de revendre le butin des vols, et de se regrouper pour organiser et perpétrer les grands coups. Le saut de frontières, qui permet aux bandits de se soustraire aux poursuites en se réfugiant dans une juridiction voisine sitôt leur méfait commis, est pratiqué uniformément par toutes les bandes de voleurs. L'absence «d'un rigoureux contrôle policier des individus» constitue une aubaine pour le milieu du banditisme, "qui tire une puissance supplémentaire d'une forte mobilité géographique» ${ }^{32}$. En tant que professionnels du crime, les bandits savent mettre à profit les failles du système répressif d'Ancien Régime, particulièrement inadapté à la poursuite des illégalismes transfrontaliers. Les difficultés de l'entraide judiciaire entre États, ajoutées à l'absence de contrôle policier et à la perméabilité des prisons, permettent souvent aux bandits d'échapper à la justice en passant les frontières: rares sont les procès où tous les prévenus sont effectivement présents lors du verdict.

\section{II. "ON N'A JAMAIS VU LE COUPABLE JOUIR IMPUNÉMENT DE SON CRIME»}

\section{L'arrestation des suspects}

Les échanges de correspondance entre juridictions, verbaux d'arrestations et autres récits d'évasions et d'actes de rébellion à justice, recueillis au fil des procédures, témoignent des difficultés que rencontre l'appareil répressif dans sa tâche de restauration et de maintien de l'ordre public. La cause de ces difficultés réside essentiellement dans l'inadéquation des moyens d'action de la justice à la spécificité de la criminalité associative. L'extraordinaire mobilité, le professionnalisme et l'organisation des bandes criminelles pose toute une série de problèmes policiers, procéduraux, politiques et pénaux qui surgissent à différents moments de la procédure, à commencer par l'arrestation des suspects.

La République de Genève ne dispose d'aucun corps de fonctionnaires spécialement affecté aux tâches de police: les soldats de la garnison, ponctuellement détachés de leur affectation habituelle en fonction des besoins, constituent la seule force de police dont disposent les magistrats pour procéder à l'arrestation des suspects. Si ces détachements peuvent intervenir relativement rapidement dans le cadre urbain, leur intervention dans les campagnes est plus problématique, en raison de la distance qui

${ }^{31}$ PC 14236, 1784, vols avec effraction, «Conclusions », folio 4.

32 Porret (1995a, p. 313). 
sépare les lieux de cantonnement de la troupe des régions rurales. Les auteurs d'actes de brigandage échappent la plupart du temps aux poursuites, protégés par leur capacité à se déplacer rapidement et à disparaître aussitôt leur forfait commis: il en va ainsi de la plupart des voleurs de grands chemins, sur lesquels la justice ne parvient que très rarement à mettre la main. Le cas du noble savoyard Copponex illustre parfaitement la difficulté d'arrêter ces criminels qui se déplacent toujours en armes et qui n'hésitent pas à en faire usage pour s'échapper. Le 12 novembre 1769, inculpé d'excès et de violences commis quelques jours plus tôt, le bandit savoyard s'échappe aussitôt après avoir été appréhendé par le Châtelain d'Avully sur ordre du Petit Conseil :

Le dimanche 12, à 3 heures après midi, Copponex se présente à Avully audit Sieur Châtelain, qui le fait arrêter et le met à la garde des Sieurs Patthey et Chappuis et de sept ou huit paysans dans le cabaret du nommé Voan.

À onze heures de la nuit, $M$. le Châtelain étant dans la chambre de Copponex, on vint lui dire qu'une troupe d'assassins venaient enlever Copponex. Il descend seul, il voit une troupe d'hommes armés qui l'environnent en lui disant avec jurements affreux, qu'on nous rende Copponex, nous le voulons mort ou en vie. Ces hommes l'enjouent avec leurs fusils; pour éviter ces coups de fusil, il se jette dans la cuisine. Il y trouve d'autres hommes armés qui l'arrêtent et lui demandent Copponex. L'un d'eux veut lui tirer un coup de fusil; un homme d'Avully l'en empêche. M. le Châtelain s'en va à l'église faire sonner le tocsin. Pendant ce temps, cette troupe tire sur les gens d'Avully, et leur fusils faisant faux feu, ils les frappent à coups de crosse. Pendant ce temps encore, de Copponex s'évade et se joint à la troupe, qui tire encore plusieurs coups de fusil sur les gens d'Avully ${ }^{33}$.

Cette évasion rocambolesque illustre parfaitement le déséquilibre des forces en présence: une milice rurale désorganisée fait bien piètre figure face à une troupe de bandits déterminés, dont la mobilité et l'habitude du maniement des armes constituent les atouts principaux.

Une fois les coupables arrêtés et réduits dans les geôles de la Seigneurie, encore faut-il parvenir à les y garder: «on s'échappait assez facilement des prisons d'Ancien Régime $»^{34}$, comme le prouvent les nombreuses notices faisant état d'évasions trouvées dans les procédures. Spécialistes de l'effraction ou du vol armé, les bandits sont également des champions de l'évasion. Une correspondance en provenance du Pays de Gex signale, par exemple, que le bandit Mathieu recherché pour plusieurs vols avec effraction commis sur sol genevois en 1781, est parvenu à s'échapper par deux fois des mains de la Maréchaussée en l'espace de six mois:

Le nommé Louis Mathieu, portant ci-devant le nom de Philippe Aucourt et connu sous celui de Grand Philippe, qui a été fouetté, marqué et banni à perpétuité des terres de Genève au mois de mai 1778, qui fut ensuite arrêté à Versoix, et qui s'échappa à Auxerre des mains des cavaliers de Maréchaussée qui le conduisaient à Bicêtre sur un ordre du Roi du 24 oct. 1779 , ayant été de nouveau arrêté à Chambéry et réclamé par la France, s'est encore évadé des prisons de Beaune où il avait été mis en dépôt pour une nuit par deux cavaliers de Maréchaussée qui le transféraient à Bicêtre sur un second ordre du Roi du 24 avril $1780^{35}$.

33 PC 11942, brigandage, rébellion, «Conclusions du procureur général Mercier», folio 1.

34 Zysberg (1987, p. 13).

35 PC 13078, vols et complicité, «Lettre du Sieur Fabri de Gex, du 30 juillet 1780 ». 


\section{L'entraide judiciaire}

Nécessaire à la répression d'une criminalité qui utilise le morcellement territorial pour tenter d'échapper à la justice, la coopération judiciaire complique singulièrement les poursuites et diminue les chances de châtier rapidement et systématiquement les bandits. L'aide des juridictions voisines est très souvent sollicitée pour arrêter les auteurs présumés d'un crime; tel est le cas, par exemple, pour Marnac et Blanchard après le vol d'un comptoir d'horlogerie en 1782. Ayant commis leur vol durant la nuit, les deux complices quittent la ville dès l'aube, au moment même où leur forfait est découvert. Le Sieur Ohrly, voisin des victimes qui connaît les suspects de vue pour les avoir vu rôder devant sa boutique la veille du vol, est chargé par la justice de se lancer à leur poursuite avec une missive demandant aux magistrats du Pays de Vaud de les arrêter. Il les rejoint à la hauteur de Rolle, mais le Châtelain du lieu refuse de procéder à l'arrestation. Le bailli de Morges se montre plus coopératif, arrête les deux suspects, les fait fouiller et les interroge. Craignant des représailles de leur part, le Sieur Ohrly refuse d'assister à l'interrogatoire, et les deux bandits sont finalement relâchés ${ }^{36}$.

Les coûts engendrés par l'entraide judiciaire (échanges de correspondances, envoi de détachements, paiement des frais de détention) et les conflits de compétence restreignent celle-ci aux cas les plus graves ${ }^{37}$. L'arrestation de suspects en terre étrangère et leur éventuelle extradition bute, en outre, sur le problème de l'identification. Lorsqu'aucun témoin oculaire n'est présent pour identifier les suspects, l'identification s'effectue par le biais de signalements, envoyés avec les «lettres rogatoires" demandant la recherche et l'arrestation d'un ou plusieurs individus. Seuls instruments dont dispose la justice pour reconnaître les individus, les signalements ne permettent pas toujours d'établir l'identité d'un suspect avec certitude; celui-ci est alors relâché, faute d'avoir pu être formellement identifié ${ }^{38}$.

Les affaires de banditisme créent fréquemment des tensions diplomatiques entre les États, qui s'accusent mutuellement de ne pas faire preuve d'assez de rigueur répressive. Dans sa lettre du 11 mars 1757 adressée au Petit Conseil, le gouverneur de Savoie s'indigne du fait que les brigands qui «assassinent et rançonnent les sujets de Sa Majesté » puissent trouver refuge sur le territoire de Genève, où ils se retirent «impunément après coup, pour entreprendre d'autres attentats à la première occasion». Ajoutant que l'on «ne saurait parvenir au but de les dissiper entièrement, si le territoire de ladite ville leur est ouvert, et s'ils y trouvent l'impunité $\gg^{39}$, il met en

36 PC 14236bis, 1784, Blanchard et consorts, "Conclusions», folio 24.

37 L'entraide judiciaire entre Genève et les juridictions environnantes (France, Savoie, Pays de Vaud, voire Neuchâtel) n'a suscité, à ce jour, aucune étude approfondie. Henry (1984), dans son étude sur la criminalité neuchâteloise, consacre une dizaine de pages au sujet, mentionnant quelques cas d'extradition, dont celui du voleur et meurtrier Montandon-dit-Clerc, extradé du Pays de Gex à Môtiers en 1742 (pp. 232-242). ll constate cependant que la «pratique de l'extradition était [...] une rareté» (p. 234).

38 Ancêtre de la fiche anthropométrique, le signalement dresse en quelques lignes le portrait d'un individu, plus ou moins circonstancié selon les cas. Envoyées d'une juridiction à une autre, dans le cadre de la coopération judiciaire, ces descriptions signalent en général les traits suivants: l'âge, la stature, la morphologie du visage, les autres signes particuliers, parfois l'habillement, l'élocution ou encore l'équipage. Á ce sujet, $c f$. Porret (1998).

39 AEG, Registres du Conseil, No. 257, «Lettre de M. Sinsan, gouverneur de Savoie du 11 mars 1757 », pp. 170-171. 
évidence la nécessité impérative d'une coopération étroite entre les juridictions, sans laquelle toute tentative de répression s'avère inefficace. Les actions d'entraide judiciaire entreprises au XVIII ${ }^{\mathrm{e}}$ siècle présentent un bilan globalement mitigé: même si elles permettent d'arrêter et de châtier quelques bandits, beaucoup d'autres réussissent à échapper aux poursuites, ou à s'évader avant d'être extradés.

\section{L'instruction du procès}

Au cours de l'instruction du procès, les Auditeurs ont la charge d'établir, en premier lieu, le corps du délit, puis de découvrir toutes les circonstances du crime qui permettront, en dernier lieu, au procureur général de qualifier le crime et de motiver la peine. Dans les procès intentés contre des criminels associés, les magistrats sont tenus de respecter scrupuleusement les mêmes règles procédurales que dans tout autre procès: il n'existe pas, à Genève, de tribunaux d'exception, comme ceux de la Maréchaussée en France, qui rendent une justice expéditive et sans appel dans toutes les affaires de vol associatif commis dans les campagnes ${ }^{40}$. La condamnation d'un prévenu doit reposer sur des preuves légales, dont l'établissement exige un long et scrupuleux travail d'enquête $e^{11}$. Les preuves que les magistrats instructeurs s'efforcent de réunir sont de plusieurs types: dépositions de témoins, aveux des prévenus, pièces à conviction (objets volés, armes, outils servant à l'effraction, etc.), flagrant délit, témoignages de complices, rapports d'experts. Au terme de l'instruction, la condamnation du prévenu ne peut intervenir que lorsque l'ensemble des pièces du procès fournit suffisamment d'éléments de certitude pour emporter la conviction des juges. Lorsque les preuves manquent, ou qu'elles ne concordent pas, le prévenu est en général relâché, ou condamné sur présomption à une peine de moindre gravité, le plus souvent un bannissement.

Dans les affaires de banditisme, l'établissement du corps du délit et de la culpabilité des prévenus rencontre des obstacles particulièrement difficiles à surmonter, en raison de la nature de l'organisation criminelle, de celle des crimes et de l'endurcissement des prévenus. En effet, les bandits connaissent d'expérience les moyens de limiter les indices dont le recoupement pourrait entraîner leur condamnation; la nature même de leurs crimes (souvent commis de nuit, dans des endroits isolés) les rend difficiles à prouver. Il n'entre pas dans notre propos de détailler les multiples problèmes qui peuvent surgir lors de l'établissement de chaque type de preuve dans toutes les procédures étudiées. Contentons-nous d'exposer de manière synthétique les principales difficultés qui ressortent des procédures prises dans leur ensemble.

L'avis des jurisconsultes diverge quant à l'importance que revêt l'aveu en tant que preuve. Jusqu'en 1738, date de l'abolition de la torture à Genève, aucune condamnation ne peut avoir lieu tant que le prévenu n'a pas avoué ses crimes. Plusieurs bandits parviennent ainsi à éviter la peine capitale en résistant à la torture. Rompus au fonctionnement de la machine judiciaire, ils savent qu'ils augmentent leurs chances d'éviter la condamnation en niant les faits qui leur sont reprochés. Dès

40 À propos de la justice expéditive rendue par les Prévôts des Maréchaux en France, $c f$. Castan (1976) et Dyonet (1997); pour la Flandre, $c f$. Lorgnier, Martinage (1981).

41 Nous n'entrerons pas ici dans le détail, par ailleurs fort complexe, du système des preuves légales: les avis mêmes des jurisconsultes divergent quant à la façon de considérer les preuves et de les classer, et les procureurs généraux n'utilisent pas unanimement la même classification. 
1738, la suppression de la torture entraîne une modification des exigences procédurales en matière de preuves. Convaincu que plus «les coupables ont commis de crimes, plus leur conscience est endurcie, plus leur négative est absolue, et par conséquent la chance d'échapper à la mort irait pour eux en croissant», le procureur général Naville stipule que l'aveu n'est pas une condition sine qua non de la condamnation des prévenus. Il doit être conforté par d'autres preuves, qui prennent dès lors une importance grandissante dans l'instruction: «des témoins irréprochables, des documents parfaitement clairs, des indices directs indubitables plus clairs que le jour, voilà les preuves admises en matière criminelle à défaut d'aveu $\gg^{42}$.

La preuve «testimoniale» est une de celles qui a le plus de force de conviction: selon le jurisconsulte genevois Sartoris (1706-1780), « deux témoins irréprochables, et uniformes dans leur déclaration ou rapport devant le Juge, suffisent pour former une preuve, qui doit être regardée comme complète $»^{43}$. Pourtant, dans les affaires de banditisme, les témoignages sont difficiles à recueillir, en raison de la crainte que les bandits inspirent. Effrayés par leur réputation et craignant pour leur vie en cas d'acquittement, les témoins hésitent à parler, encore plus à soutenir leurs accusations lors des confrontations avec les prévenus. Plusieurs procureurs généraux déplorent ce fait, qui accrôt la difficulté des poursuites: «la crainte d'être la victime de leur fureur, fait que l'on n'ose ni se plaindre, ni concourir par des indications ou des témoignages à délivrer la société de ces scélérats $»^{44}$. Dans l'affaire du vol Binet et Miroglio en 1782, déjà évoquée plus haut, les deux suspects sont relâchés par le Châtelain de Morges parce que le seul témoin capable de les confondre, le Sieur Ohrly, refuse de leur être confronté, craignant pour sa sécurité: «s'ils m'avaient connu comme leur accusateur, ma vie aurait été en danger», avoue-t-il à l'Auditeur lors de sa déposition ${ }^{45}$.

Le flagrant délit, une des preuves les plus convaincantes de culpabilité, est rare dans les affaires de banditisme: lors de brigandages, de vols avec effraction ou usage de fausses clefs, ou même de filouteries, la mobilité des voleurs, leur discrétion et leur organisation, la protection des ténèbres, leur permettent dans la plupart des cas de ne pas être pris sur le fait.

Les pièces à conviction - objets ou effets volés, armes et outils d'effraction (fausses clefs, cire pour les empreintes de serrures, rossignols) - forment la dernière grande catégorie de preuves sur lesquelles les magistrats peuvent se fonder pour démontrer la culpabilité des prévenus. Cependant, une fois encore, les bandits connaissent le danger d'être appréhendés en possession de ces objets compromettants, et ils disposent de moyens efficaces de faire disparaitre toutes traces de leurs méfaits. Le produit des vols est rapidement mis hors d'atteinte de la justice grâce aux réseaux de receleurs, ou dissimulé immédiatement après le vol. Quant aux outils d'effraction et aux armes, ils sont laissés en dépôt chez des complices ou cachés dans un repaire. En dernier recours, les bandits tentent de se débarrasser des objets compromettants juste avant d'être arrêtés: tel est le cas, par exemple, des bijoux

\footnotetext{
42 PC 14236bis, 1784, Blanchard et consorts, «Conclusions», folio 61.

43 (1773, II, pp. 432-433).

44 PC 9710,1750 , vols et brigandage, "Conclusions du procureur général Buisson», folio 48.

45 PC 14447, vols, complicité de vol, «Déposition de Jean-Pierre Ohrly».
} 
volés par Marnac et Blanchard chez Binet et Miroglio, qu'ils jettent sous une voiture alors qu'ils sont escortés au château de Morges en $1782^{46}$.

La plus grande difficulté rencontrée par les magistrats découle de la grande mobilité géographique des bandits, qui commettent leurs méfaits sur des territoires dépendant de plusieurs juridictions différentes. Même si, en principe, les juges genevois peuvent connaître des délits commis en terres étrangères, pour autant que l'accusé soit détenu dans les prisons de la Seigneurie, l'instruction de tels procès se révèle toujours complexe, voire impossible. La condamnation du prévenu doit en effet se fonder sur des preuves suffisantes, preuves difficiles à réunir lorsqu'aucun témoignage direct n'est disponible. Le procureur général subrogé Des Franches, amené à motiver les peines de cinq membres d'une bande de filous en 1765, abandonne ainsi les charges qui pèsent contre eux pour des crimes commis dans une autre juridiction: «les délits antérieurs à la procédure n'ont pas été commis sur notre territoire; et qu'ignorant leurs circonstances et leurs détails, ils ne peuvent mériter de la part de Vos Seigneuries la même animadversion ${ }^{47}$. Dans les cas où des crimes commis hors des frontières de la République constituent les seules charges retenues contre un prévenu, celui-ci est généralement relaxé faute de preuves. Arrêté pour fréquentations suspectes en 1788 , Claude Mérite, surnommé Chavanel, est relâché après deux mois de détention, malgré sa qualité de récidiviste, prouvée à la fois par une expertise chirurgicale, et par une lettre de Lyon le présentant comme «un fameux brigand qui s'était sauvé sept à huit fois des galères et qui était un homme des plus dangereux ${ }^{48}$.

\section{Le châtiment des scélérats}

Dans son Examen des causes de l'augmentation du nombre des brigands, publié en 1751, l'anglais Henry Fielding analyse les causes de l'échec de la répression du banditisme dans l'Angleterre du XVII' ${ }^{\circ}$ siècle. Cet ancien magistrat, rompu à l'exercice de la justice par une longue pratique des prétoires, souligne, entre autres, la rareté des dénonciations, les difficultés liées à la réunion des preuves nécessaires à la condamnation des prévenus, l'impunité des receleurs, la publicité des châtiments qui entraîne la sympathie du public pour les suppliciés, les grâces trop fréquemment accordées, et enfin «la probabilité d'échapper au châtiment ${ }^{49}$. Nous avons déjà constaté que l'appareil répressif genevois rencontre effectivement plusieurs de ces difficultés dans la poursuite des bandes criminelles. Il reste maintenant à savoir si les coupables échappent effectivement aux châtiments, comme peuvent le laisser croire les multiples indices relevés ci-dessus, et si ce fait peut être regardé comme une cause, si ce n'est de l'augmentation des actes de banditisme, tout au moins de la permanence de ce phénomène.

La criminalité apparente englobe l'ensemble des vols associatifs dont les archives judiciaires de la République portent la trace, y compris tous ceux dont les

46 PC 14236bis, 1784, Blanchard et consorts. En l'occurrence, le paquet contenant trente-cinq paires et demie de boucles d'oreilles est retrouvé par la suite, et sert de pièce à conviction dans le procès de Marnac. Celui-ci porte en effet à l'oreille la boucle manquante lors de son arrestation l'année suivante, indice qui servira de preuve à charge contre lui lors de son procès. ("Conclusions", folio 31). PC 11359, vol [filouterie], «Conclusions», folio 42.

48 PC 15395, association avec une bande de voleurs, «Verbal de l'Auditeur Saladin».

49 (1990 [1751], p. 141). 
auteurs sont restés impunis. Tel est le cas des vols de grand chemin et des actes de brigandage, dont les auteurs échappent la plupart du temps à la justice: ces vols font le plus souvent l'objet d'une simple information, sans donner lieu à une procédure faute d'éléments suffisants pour lancer une enquête. Il faudrait, pour pouvoir analyser avec précision la criminalité associative apparente, parvenir à dénombrer exactement le nombre d'«informations» se rapportant à des actes de banditisme sur l'ensemble du siècle. Une telle recherche ne donnerait que des résultats partiels et peu fiables, compte tenu des renseignements très sommaires et lacunaires fournis par ces «informations». Les chiffres ci-dessous ont été calculés à partir des quelques éléments, souvent incomplets ou sujets à caution, fournis par les procédures et informations que nous avons dépouillées.

Le nombre de criminels impliqués dans ces crimes restés impunis s'élève à 33; si on ajoute à ce chiffre les quinze jugés par contumace, le nombre de bandits ayant échappé à toute punition effective à Genève s'élève alors à 48 , soit plus de la moitié des bandits figurant dans les procédures et informations $(86)^{50}$.

Tableau 1: Criminalité réprimée et criminalité apparente

\begin{tabular}{|c|c|c|c|c|}
\hline $\begin{array}{c}\text { Bandits jugés } \\
\text { et condamnés } \\
\text { (contumaces } \\
\text { exceptées) }\end{array}$ & $\begin{array}{c}\text { Bandits jugés } \\
\text { et condamnés par } \\
\text { contumace }\end{array}$ & $\begin{array}{c}\text { Bandits jugés } \\
\text { et relâchés } \\
\text { ou extradés }\end{array}$ & $\begin{array}{c}\text { Bandits } \\
\text { non jugés } \\
\text { (proclamés ou } \\
\text { non identifiés) }\end{array}$ & $\begin{array}{c}\text { Total } \\
\text { des bandits } \\
\text { apparaissant } \\
\text { dans les PC }\end{array}$ \\
\hline 29 & 15 & 9 & 33 & 86 \\
\hline $34 \%$ & $17 \%$ & $11 \%$ & $38 \%$ & $100 \%$ \\
\hline
\end{tabular}

Il ne faut pas oublier, cependant, que la criminalité apparente ne représente pas, loin s'en faut, la criminalité réelle, qu'il est impossible de chiffrer faute de base statistique objective. Quelques indices laissent cependant imaginer son ampleur: le plus convaincant est sans doute le nombre des complices non inculpés que les bandits dénoncent lors de leur procès. Comtois le Rouge, membre de la Grande Bande, fournit à la justice les noms de 44 complices de sexe masculin ayant des relations plus ou moins régulières avec la bande (si l'on inclut les complices de sexe féminin, ce chiffre passe à 73); François Rosay, quant à lui, donne plus de vingt noms ${ }^{51}$. Ces indications, sans fournir aucune preuve objective, autorisent cependant à postuler l'existence d'un nombre indéterminé de crimes, commis par des complices, qui n'apparaissent pas dans les archives criminelles de la République, ni probablement en totalité dans les sources de la pratique pénale d'autres juridictions. Le chiffre de 48 bandits sur 86 échappant à tout châtiment effectif, calculé sur la base statistique de la criminalité apparente, se situe donc, selon toutes probabilités, bien en deçà du nombre réel de bandits impunis.

Les archives judiciaires fournissent en revanche des données plus précises sur la criminalité réprimée, que l'on peut quantifier d'une manière plus fiable. La statis-

\footnotetext{
50 Le chiffre de 86 ne comprend que les criminels ayant pris une part active à un délit: il ne comptabilise pas les complices dont la culpabilité est moindre.

st PC 14236, et PC 15312/15313.
} 
tique des jugements prononcés contre les 55 bandits sélectionnés pour ce travail montre que, sur les 53 jugements rendus par la justice genevoise entre 1682 et 1792 (le fait que quelques bandits sont jugés à plusieurs reprises, et que d'autres ne passent pas en jugement explique le chiffre de 53 jugements pour 55 bandits), 44 sentences sont prononcées, et neuf criminels sont relâchés ou extradés vers une autre juridiction. Sur ces 44 sentences, quinze sont prononcées contre des criminels contumax, soit à peu près un tiers des condamnations ${ }^{52}$.

Tableau 2: Sentences prononcées

\begin{tabular}{|c|c|c|c|c|c|}
\hline Pendaison & Roue & Bannissement & Galères & Détention & Total \\
\hline 21 & 3 & 18 & 1 & 1 & 44 \\
\hline
\end{tabular}

Tableau 3: Relaxes, extraditions et contumaces

\begin{tabular}{|c|c|c|}
\hline Relaxes & Extraditions & Contumaces (sur 44) \\
\hline 5 & 4 & 15 \\
\hline
\end{tabular}

Sur le plan de la criminalité réprimée, 24 bandits sur 53 , soit un peu moins de la moitié, échappent en définitive à la punition de leurs crimes, en tous cas sur territoire genevois.

L'analyse quantitative des sources de la pratique pénale montre que la criminalité associative subit des fluctuations, surtout à partir du milieu du XVIII' siècle. Le nombre de procédures ouvertes contre des bandits augmente fortement dans la seconde moitié du siècle: alors qu'on n'en dénombre que quatre entre 1682 et 1748 , le chiffre passe à 25 pour la période $1749-1792^{53}$. Ces chiffres doivent cependant être analysés avec précaution. Le nombre global de procédures et d'informations criminelles augmente en effet considérablement entre le début du siècle et les dernières années de l'Ancien Régime. Michel Porret en dénombre 725 pour les années 1720-1727; ce chiffre passe à 2281 pour les années $1783-1789$, soit à peu près trois fois plus que le premier ${ }^{54}$. En tenant compte de cette augmentation générale du nombre de procédures, due à une poursuite plus systématique des crimes, la criminalité associative réprimée à Genève dans la seconde moitié du XVIII ${ }^{e}$ siècle représente presque le double de celle recensée pour la première moitié du siècle.

De nombreux éléments peuvent expliquer une telle augmentation. Les causes structurelles du banditisme sont difficiles à circonscrire précisément, comme l'a fait remarquer l'historienne hollandaise F. Egmond: «la pauvreté, les guerres, l'immi-

52 Notre statistique est fondée sur les sentences définitives, rendues par le Petit Conseil, ou prononcées par le Conseil des Deux-Cents (instance seule habilitée à juger des recours en grâce) dans les cas où un recours a été accepté.

53 Pour le calcul de ces chiffres, nous n’avons comptabilisé que les procédures ayant abouti à un jugement ou à un non-lieu. Les archives judiciaires comprennent plusieurs procédures se rattachant à la même bande ou au même bandit. Dans de tels cas, nous n'avons pas comptabilisé tous les PC, mais seulement ceux qui débouchent sur un procès.

54 (1995a, p. 474). 
gration et l'exclusion sociale, combinées de diverses manières, contribuaient toutes fortement à la formation de groupes, bandes et réseaux impliqués dans le crime rural organisé " ${ }^{55}$. Les transformations socio-économiques que connaît Genève au XVIII ${ }^{e}$ siècle - urbanisation, croissance démographique, proto-industrialisation, augmentation générale du volume des échanges commerciaux et financiers - peuvent être considérées comme des causes, en tous cas partielles, de l'accroissement de la criminalité en général ${ }^{56}$. La prospérité entraîne la marginalisation d'une frange grandissante de la population; l'étalage des richesses suscite la convoitise. Suivant cette hypothèse, l'augmentation du nombre des procédures intentées contre des bandits suit logiquement la hausse générale du taux de criminalité.

Au delà des causes socio-économiques, des raisons liées plus étroitement à la criminalité entrent en ligne de compte. L'évolution du type de crimes commis par les bandits, liée à ces transformations, fournit sans doute une partie de l'explication. Les brigandages deviennent de moins en moins fréquents à mesure que la fin du siècle approche, alors que les vols avec effraction ou fausses clefs se font toujours plus nombreux. Cette hausse des crimes commis dans un cadre urbain peut rendre compte de l'augmentation du nombre de procédures ouvertes contre des bandits: alors que la poursuite des brigands sévissant dans les campagnes se révèle très difficile, le territoire urbain, plus restreint et entouré de murs d'enceinte, permet un meilleur contrôle policier des individus. Une autre cause, plus directement liée à la pénalité, doit être considérée: l'inadéquation du système pénal à la répression des bandes criminelles.

\section{L'élimination ou la récidive}

Comme le montrent les statistiques, le banditisme entraîne un renforcement de la sévérité des châtiments: en effet, sur les 44 sentences rendues contre des bandits (y compris les contumaces), 24 sont capitales. Le taux de peines capitales (55\%) prononcées dans les affaires de banditisme excède de $25 \%$ celui des sentences de mort délivrées dans l'ensemble des procès - tous crimes confondus - pour la période $1755-1781(30 \%)^{57}$. La peine de mort par pendaison est la plus couramment appliquée; seuls trois bandits sur 24 sont condamnés au supplice de la roue en $1728^{58}$. Le reste des sentences se partage entre le bannissement à perpétuité (18 sentences) accompagné ou non de fustigation et de la marque au fer rouge, la condamnation aux galères (1) et l'emprisonnement à vie (1).

Ces chiffres confirment donc le renforcement de la sévérité des peines dans les affaires de banditisme, même si ce constat doit être relativisé: quelques recours en grâce débouchent sur des commutations de peines ${ }^{59}$. En règle générale, les juges

55 Egmond (1993, p. 185). Nous traduisons de l'anglais: «In varying combinations, poverty, warfare, immigration and social exclusion all strongly contributed to the formation of groups, bands, networks involved in organized rural crime».

56 Henry (1984) signale également une forte augmentation de la criminalité réprimée au XVIII siècle à Neuchâtel; il attribue cette augmentation principalement à la croissance économique et démographique (p. 703).

57 Porret (1995a, p. 477).

58 PC 7563, larcins. Ces trois soldats de la garnison, coupables de vols avec effraction, sont par ailleurs les derniers criminels condamnés par la justice genevoise à subir cette peine particulièrement sévère.

59 Dans les affaires de banditisme, les recours en grâce sont rares: ils ne concernent qu' un prévenu sur dix. 
genevois font preuve d'une moins grande rigueur dans l'application des peines que leurs confrères des États voisins, pratique relevée par le procureur général subrogé Des Franches en 1765: «Vos Seigneuries modifient presque toujours la sévérité de la jurisprudence des autres États $»^{60}$.

La justice criminelle d'Ancien Régime est extrêmement démunie dans le traitement de la récidive, trait caractéristique de la criminalité associative. L'arsenal des peines n'offre aux juges genevois qu'une palette très limitée de châtiments. Les peines applicables aux délinquants récidivistes coupables de crimes «atroces» sont au nombre de trois seulement: la peine capitale (exceptionnellement sur la roue, en général par pendaison), les galères (à temps ou à perpétuité) et le bannissement (accompagné ou non de fustigation et de marque au fer rouge). L'emprisonnement, seule peine capable de garantir la sécurité de la société tout en laissant la vie sauve au criminel, n'est en effet utilisé au XVIII ${ }^{e}$ siècle que de manière préventive pendant l'instruction des procès. La détention ne figure pas dans le catalogue des peines établi par l'Ordonnance de 1670, et n'est prononcée qu'à titre exceptionnel : seul le noble Copponex, suite à un recours en grâce et aux pressions des autorités savoyardes, bénéficie de ce traitement de faveur en 1776.

La condamnation aux galères qui répond, en principe, à la nécessité de retrancher un criminel de la société, est étonnamment peu utilisée au cours du XVIII ${ }^{\mathrm{e}}$ siècle, probablement en raison des coûts qu'elle engendre et de la nécessité d'arrangements politiques qu'elle nécessite avec les États (Piémont et France) qui disposent de lieux de détention pour les galériens, à défaut d'une flotte de galères à proprement parler (en France, la flotte est définitivement laissée à quai dès 1748). L'absence de concordat entre la République et ces deux pays, pendant un laps de temps inconnu, fournit l'explication la plus plausible de la rareté des peines de galères $^{61}$. Qui plus est, l'enfermement aux galères ne garantit pas forcément la neutralisation des criminels. Quelques-uns des bandits prévenus de crimes à Genève sont d'anciens galériens en fuite; d'autres, condamnés à temps, ont été libérés après avoir purgé leur peine; d'autres, enfin, ont obtenu la remise de leur peine après s'être engagés à servir dans les armées royales dont ils ont déserté, ou ont obtenu leur libération par corruption ${ }^{62}$.

Le bannissement, sentence la plus souvent prononcée contre les bandits après la peine de mort, n'atteint pas le but premier qu'il recherche, à savoir la protection de la société, comme le relèvent nombre de pénalistes dans la seconde moitié du XVII' siècle. Le réformateur de la justice criminelle Brissot de Warville (1754-1793), parmi d'autres, dénonce l'inutilité du bannissement et appelle de ses vœux des peines d'emprisonnement capables de protéger la société de ses éléments les plus dangereux: «Lorsqu'un chien enragé menace de communiquer à tous les autres animaux le poison dont il est attaqué, suffit-il donc de l'éloigner, de le bannir d'un canton, lorsqu'on peut préserver les endroits voisins de la contagion qu'il peut

\footnotetext{
60 PC 11359, vol, «Conclusions», folio 41.
}

61 Sur l'ensemble du siècle, quatre bandits seulement sont condamnés à cette peine, et trois d'entre eux bénéficient d'une commutation de leur peine après avoir recouru en grâce en 1769 (PC 11942). À notre connaissance, aucune étude n'a jusqu'ici été menée sur ces concordats, qui permettrait de connaître avec précision la période de carence de tels accords. La seule certitude concerne la date de 1782 , à laquelle un nouveau concordat entra en vigueur entre Genève et la France.

62 D' après Zysberg(1987), seul 1\% des détenus s'évade réellement des galères. Ce chiffre, cependant, ne comprend pas les évasions «subtiles», par corruption ou enrôlement, beaucoup plus nombreuses. 
répandre ? $^{63} \gg$. Le bannissement, en effet, ne sert qu'à déplacer géographiquement le danger dans les contrées environnantes, sans l'éliminer: les criminels bannis d'une juridiction s'en vont poursuivre leurs activités dans les États voisins. Bien souvent, même, les bandits bannis de la République reviennent commettre de nouvelles exactions sur le territoire genevois quelques temps plus tard. Encourageant indirectement la récidive, le bannissement «fait de la justice la pourvoyeuse même du banditisme $»^{64}$. Son inefficacité peut donc être considérée comme une des causes, si ce n'est de l'augmentation, du moins de la permanence des actes de banditisme.

Dernière peine à disposition des juges et seule à garantir l'élimination définitive du danger que les criminels récidivistes représentent pour la société, la peine de mort est le châtiment le plus fréquemment appliqué aux bandits. Au XVIII siècle, pourtant, les juges genevois rechignent à infliger cette peine qui, si elle présente l'avantage de retrancher définitivement les fauteurs de troubles de la société, a l'inconvénient de ne laisser aucune place à l'éventuelle régénération du criminel. Or, durant la seconde moitié du siècle, «le souci d'amender le condamné par la peine ${ }^{65}$, est de plus en plus présent à l'esprit des magistrats de la République. Ils ne prononcent la peine capitale que dans les cas les plus graves, lorsque l'atrocité des crimes l'exige et que les preuves de l'incorrigibilité du délinquant sont suffisamment probantes pour leur faire abandonner tout espoir d'amendement par une peine moins sévère.

Le réquisitoire rédigé en 1787 par le procureur général subrogé Rigaud contre le bandit Bassière, dit Courtebotte, illustre parfaitement le dilemme soulevé par la punition des criminels récidivistes. Auscultant minutieusement la carrière criminelle du bandit, le magistrat s'attache d'abord à prouver l'échec des peines auxquelles il a été précédemment condamné à susciter chez lui un quelconque repentir:

ne témoignant ni repentir ni remords, il ne permet pas même à ses juges de chercher à le sauver par l'idée d'une régénération, dont les tribunaux doivent toujours avoir l'espoir, tant que cette espérance n'est pas démontrée chimérique ${ }^{66}$.

Convaincu de la nécessité de retrancher définitivement le fauteur de troubles de la société et de l'empêcher ainsi de commettre de nouveaux crimes, le procureur général requiert finalement la peine capitale:

S'ils [les juges] rendent Bassière à la société, elle dira: vous rejetez dans mon sein un homme qui n'y a vécu que de brigandages et de rapines. Vous connaissez son penchant invincible au mal. Vous savez avec quels scélérats il est associé. L'exemple de Souche son compagnon [condamné quelques temps auparavant à une peine infamante] vous montre ce que vous devez attendre de ces supplices dont l'infamie, nulle pour de tels scélérats, et la douleur passagère, en les perdant pour jamais ne les rend que plus hardis à tout entreprendre par l'espoir toujours si séduisant d'échapper à la corde et qui s'établissent à nos portes pour y continuer leurs brigandages.

[...] Quand les Athéniens mirent à mort ce jeune homme qui se plaisait à crever les yeux de ses oiseaux, ils songèrent moins à le punir de sa cruauté

\footnotetext{
63 Buissot de Warville (1782, pp. 186-187).

64 Castan (1986, p. 114)

65 Roth (1992, p. 166).

${ }_{66}$ PC 15159 , vols et assassinats, «Conclusions », folio 49.
} 
qu'à se préserver des attentats plus atroces dont cette barbarie leur parut un présage indubitable ${ }^{67}$.

Signe de la clémence des juges genevois, le Petit Conseil refuse de suivre le réquisitoire du procureur général et condamne le bandit à un bannissement des terres de la Seigneurie, accompagné de fustigation et de la marque au fer ardent.

Le souci de donner au délinquant la possibilité de s'amender se heurte toujours à la nécessité d'assurer l'ordre public et de garantir la sécurité des citoyens. Tiraillés entre ces deux exigences et ne disposant pas d'autre alternative, les juges n'optent pour la seconde que lorsque le parcours criminel du prévenu présente suffisamment d'indices d'un endurcissement irréversible et que l'atrocité de ses crimes l'exige. Plusieurs voix s'élèvent pourtant, dans la seconde moitié du XVIII' siècle, pour réclamer des peines d'enfermement, comme celle de l'avocat Du Roveray en 1771:

[O]n ne saurait assez regretter de ne pas avoir chez nous ces établissements publics au moyen desquels la société se purge des membres qui la déshonorent, sans en infecter les États voisins, et où les criminels, subissant tous les jours une peine exemplaire et corrective, remplissent ainsi le vrai but des châtiments ${ }^{68}$.

Néanmoins, avant l'établissement de la détention carcérale en 1825 à Genève, l'absence d'une peine intermédiaire efficace en termes d'utilité sociale, entre la peine de mort et le bannissement, ne laisse aux juges que deux possibilités : l'élimination définitive du délinquant, ou le bannissement qui équivaut pratiquement à encourager la récidive. L'impossibilité de neutraliser un individu dangereux par d'autres moyens que son élimination physique, autrement dit l'inefficacité de la pénalité d'Ancien Régime dans le traitement de la récidive, explique le taux élevé de peines capitales prononcées dans les affaires de banditisme, et doit être mise au nombre des causes de la permanence du banditisme, «fléau» endémique des sociétés modernes. Ces causes, liées à la procédure et à la pénalité, ne doivent cependant pas faire oublier les problèmes sociaux et structurels qui font le lit du banditisme: les conditions socio-économiques du XVIII ${ }^{e}$ siècle, qui génèrent le déracinement et la pauvreté dans toute une frange de la population, contribuent largement à renouveler le contingent des exclus pour qui l'association criminelle constitue avant tout un moyen de subsister.

\section{CONCLUSIONS}

L'étude du banditisme à Genève au XVIII ${ }^{e}$ siècle confirme, dans la perspective d'une histoire de la criminalité, l'évolution observée dans un grand nombre de pays européens: les crimes de sang décroissent au profit de la criminalité acquisitive. Les agressions de voyageurs ou d'habitants des campagnes cèdent le pas, peu à peu, à des techniques de vol plus élaborées, telles que l'effraction, l'utilisation de fausses clefs ou encore la filouterie sous toutes ses formes. Sur le plan spatial, les bandes criminelles investissent le cadre urbain, délaissant progressivement, surtout à partir du

67 PC 15159, «Conclusions», folios 46-47.

68 PC 12149, «Requête de Du Roveray ». À propos de l'histoire de la réforme pénale et de la naissance de la prison à Genève, $c f$. Roth (1981) et Porret (1995a; 1997); pour la France, $c f$. Petit (1990). 
milieu du siècle, le grand chemin et les habitations isolées pour se concentrer sur les réservoirs de richesses de la cité. Cette évolution ne doit pas être comprise comme un changement drastique, mais bien plutôt comme une tendance généralisée: à côté des bandes de voleurs professionnels sévissant en ville subsistent quelques troupes de brigands, composées de déserteurs de passage ou d'individus en rupture de la société paysanne des régions frontalières de Genève.

Le modèle du «bandit social» proposé par Hobsbawm et utilisé par de nombreux historiens dans son sillage, qui considère le banditisme comme un «phénomène rural dans des sociétés essentiellement agraires ${ }^{69}$, se révèle donc inadéquat, ou tout du moins insuffisant, en tant que paradigme d'analyse de la criminalité associative genevoise au Siècle des Lumières. La figure du «bandit d'honneur », avatar du bandit social dont les actes traduisent une révolte ouverte contre un pouvoir qu'il défie par ses crimes, redistributeur de richesses injustement spoliées à la masse désarmée des petites gens, ne correspond pas non plus à la réalité livrée par les procès criminels.

Socialement, les bandits appartiennent, à de rares exceptions près, à la cohorte des déracinés et des errants en tous genres, chassés de leurs pays d'origine par la misère, ou suite à des démêlés avec la justice. Pour ces marginaux déjà bien engagés dans la "carrière du crime», exclus de leur milieu social d'origine, l'association avec d'autres récidivistes s'impose d'elle-même: ils recréent dans les bandes les réseaux de solidarité indispensables à leur survie, et y trouvent les appuis nécessaires à la réalisation de leurs activités criminelles. Regroupés dans les faubourgs des villes, dont la croissance au XVIII ${ }^{\mathrm{e}}$ siècle suit l'évolution d'une économie en voie d'industrialisation, accompagnés de leurs femmes ou concubines, parfois de leurs enfants, ils s'adjoignent les services de quelques receleurs disposant d'une assise dans la société locale, et de plusieurs complices occasionnels issus du même milieu qu'eux. L'organisation de ces associations criminelles rappelle sous plusieurs aspects celle des corporations de métiers, dotées d'une hiérarchie et d'un langage propres (l'argot des galères), dans lesquelles les tâches sont distribuées entre chaque membre selon ses capacités.

Révélateur des modifications structurelles de la société et des tensions sociales, le banditisme dévoile également les failles du système répressif d'Ancien Régime, dont «les deux plus grandes faiblesses » sont «l'absence de l'instruction policière et le cloisonnement des espaces où peuvent s'exercer les poursuites ${ }^{70}$. Le découpage du territoire en juridictions multiples, les conflits de compétence qui surgissent parfois entre celles-ci, l'imprécision des moyens d'identification et la lenteur des communications freinent l'entraide judiciaire. Ajoutées à cela, l'absence - ou l'insuffisance - d'un corps de police permettant un contrôle serré des frontières et du territoire, ainsi que les autres difficultés rencontrées par la justice dans la poursuite des bandes criminelles (établissement des preuves, détention), expliquent en partie la permanence du phénomène du banditisme dans les sociétés modernes. Cette permanence doit aussi être recherchée dans les caractéristiques intrinsèques de la pénalité d'Ancien Régime, particulièrement désarmée pour traiter le problème de la récidive: en dehors de la peine capitale, seule capable d'éliminer définitivement la menace représentée par les bandits, la justice criminelle ne dispose d'aucun châti-

70 Dyonet (1997, p. 206). 
ment qui soit en mesure à la fois de retrancher le criminel dangereux de la société et de le corriger. La dureté de la répression (plus de la moitié des peines prononcées contre les bandits sont capitales) s'explique donc, non seulement par «l'atrocité» des crimes, mais également par l'absence de peines de substitution.

Le banditisme, qui cumule les circonstances les plus aggravantes et met en échec les garants de l'ordre public, «représente à coup sûr la forme la plus redoutable des activités criminelles ${ }^{\prime 1}$. Preuves en sont, encore, les peurs qu'il suscite et les images qu'il évoque dans l'imaginaire des couches dominantes de la population, révélées par les biographies criminelles et la littérature à l'usage du peuple retraçant la vie et les aventures de bandits célèbres ${ }^{72}$. Les représentations sociales du bandit et l'insécurité qu'il génère, constitutifs de sa dangerosité, produisent une exigence de répression accrue qui intervient à deux niveaux. Pénalement, elle se traduit par une aggravation de la qualification des crimes et une grande sévérité des châtiments; en termes législatifs, par la promulgation de lois qui marginalisent et enferment les pauvres et les vagabonds dans des lieux clos et surveillés (dépôts de mendicité, maisons de discipline, plus tard prisons). La présence du pauvre, de l'errant, tolérée encore au Moyen Âge comme une partie intégrante de la société, devient peu à peu inacceptable. «Paradoxalement, le Siècle des Lumières, que nous admirons, qui œuvre pour la défense et la reconnaissance de l'individu, donne le signal de la mise à l'écart des déviants " ${ }^{73}$.

La répression du banditisme, d'un point de vue social et politique, révèle l'affermissement de l'État en tant que garant de la sécurité publique. L'extension du contrôle social, la constitution des Etats-nations et le contrôle des frontières qui y est lié, la naissance de la prison, conjointement à l'évolution structurelle de la société (exode rural, urbanisation, développement industriel) entraîneront au XIX ${ }^{\mathfrak{e}}$ siècle, un recul du banditisme rural en Europe occidentale, qui disparaîtra presque complètement, à l'exception de certaines zones méditerranéennes ${ }^{74}$. Le cas genevois confirme cette thèse. Le banditisme urbain, en revanche, trouvera dans ces modifications de puissants stimulants: on assistera dès lors à l'émergence d'un véritable «milieu», favorisé par la croissance des villes et la promiscuité carcérale.

Christophe DUBIED Rue de l'Hôpital 7 CH-2114 FLEURIER/NE c_dubied@hotmail.com

71 Castan (1980, p. 223).

72 Les dimensions du présent article ne nous ont pas permis de développer la réflexion sur l'imaginaire social du monde criminel dans les sociétés d’Ancien Régime. Cet aspect a, en revanche, été exploré plus en détail dans notre mémoire de licence.

73 «Postface» de C. Borgella in Lorédan (1995, p. 309). La modification de la perception sociale et de la répression pénale de la pauvreté a été abondamment documentée, entre autres par Geremek (1987) et Gutton (1971).

74 Cf. Hobsbawm (1965). 


\section{RÉFÉRENCES}

Beattie J. M., Crime and the Courts in England, 1660-1800, Princeton, New Jersey, 1986. Berchtold J., Porret M., La peur au XVIII siècle: discours, représentations, pratiques, Genève, 1994.

Brissot de Warville J.-P., Théorie des lois criminelles in, Bibliothèque philosophique du législateur, du politique, du jurisconsulte, Berlin, Paris, Lyon (10 vol.), t. I, 1782.

Castan N., La justice expéditive, Annales ESC, 1976, pp. 331-361.

Castan N., Les criminels de Languedoc: les exigences d'ordre et les voies du ressentiment dans une société pré-révolutionnaire (1750-1790), Toulouse, Presses de l'Université Le Mirail, 1980.

Castan N., Du banditisme à la révolte populaire: l'exemple du Vivarais à la fin de l'Ancien Régime, in, Ortalli G. (éd.), Bande armate, banditi, banditismo (...), Rome, Jouvence, 1986.

Dufour A., Histoire de Genève, Paris, PUF, 1997.

Dyonet N., Les paroles et les écritures. Fonctionnement et bénéfices de la procédure inquisitoire en France au XVIII' siècle, Déviance et Société, 1987/3, pp. 225-249.

Dyonet N., La maréchaussée et la culture judiciaire française au temps de Beccaria, in, Porret M. (éd.), Beccaria et la culture juridique des Lumières, Genève, 1997, pp. 197-214.

Egmond F., Underworlds: Organized Crime in the Netherlands, 1650-1800, Cambridge, 1993.

Fielding H., Examen des causes de l'augmentation du nombre des brigands, [1751], Paris, Éd. des Cendres, 1990.

Funck-Brentano F, Les brigands, Paris, Hachette, 1924.

Geremek B. (Dir.), Truands et misérables dans l'Europe moderne (1350-1600), (Archives), Paris, Gallimard, 1980.

Geremek B., La potence et la pitié. L'Europe et les pauvres du Moyen Âge à nos jours, Paris, 1987.

Gutton J.-P., La société et les pauvres: l'exemple de la généralité de Lyon, 1543-1789, Paris, Gallimard, 1971.

Henry P., Crime, justice et société dans la principauté de Neuchâtel au XVIII siècle (17071806), Neuchâtel, La Baconnière, 1984.

Hobsbawm E. J., Primitive Rebels. Studies in Archaich Form of Social Movement in the 19th and 20th centuries, New York, Praeger, 1965.

Hobsbawm E. J., Les bandits, Paris, Maspero, 1972.

Johansen J. C. V., Stevnborg H., Hasard ou myopie. Réflexions autour de deux théories de l'histoire du droit, Annales ESC, 1986, pp. 601-624.

Jousse D., Traité de la justice criminelle de France, Paris (4 vol.), 1771.

Lorédan J., La grande misère et les voleurs au XVIII siècle: Marion du Faouët et ses associés, 1740-1770, Le Faouët, 1995.

Lorgnier J., Martinage R., Procédure criminelle et répression devant la Maréchaussée de Flandres (1679-1780), Revue historique de droit français et étranger, 1981, pp. 183-197.

Muyart de Vouglans P.-F., Institutes au droit criminel, ou principes généraux sur ces matières, suivant le droit civil, canonique, et la jurisprudence du Royaume; avec un Traité particulier des crimes, Paris, 1757.

Ortalli G. (éd.), Bande armate, banditi, banditismo e repressione di giustizia negli stati europei di antico regime, Actes du colloque de Venise (1985), Rome, Jouvence, 1986.

Petit J.-G., Ces peines obscures. La prison pénale en France, 1780-1875, Paris, Fayard, 1990. 
Porret M., Le crime et ses circonstances: de l'esprit de l'arbitraire au Siècle des Lumières selon les réquisitoires des procureurs généraux de Genève, Genève, 1995.

Porret M., La biographie des scélérats, ou les circonstances de la dangerosité criminelle durant l'Ancien Régime, Traverses, 1995b, 2, pp. 55-64.

Porret M. (éd.), Beccaria et la culture juridique des Lumières, Genève, Droz, 1997.

Porret M., «Signalement», «portrait parlé », cliché judiciaire: le visage des scélérats, Images, 1998, 4, pp. 34-41.

Roth R., Pratiques pénitentiaires et theorie sociale. L'exemple de la prison de Genève (18251862), Genève, Droz, 1981.

Roth R., Réformes du droit pénal à Genève durant l'époque révolutionnaire, in, Mémoires et documents publiés par la Société d'histoire et d'archéologie de Genève, Genève, 1992, 55, pp. 151-167.

Sartoris J.-P., Éléments de la procédure criminelle suivant les ordonnances de France, les Constitutions de Savoie, et les Édits de Genève, Amsterdam (2 vol.), 1773.

Serpillon F, Code criminel ou Commentaire sur l'Ordonnance de 1670, Lyon (2 vol.), 1767.

Weisser M., Crime and Punishment in Early Modern Europe, Brighton, Harvester Press, 1982, (2nd edition).

Zysberg A., Les galériens. Vies et destins de 60000 forçats sur les galères de France, 1680 1748, Paris, Seuil, 1987. 